\title{
Quadruple Coincidence and Common Quadruple Fixed Point for Hybrid Pair of Mappings Under New Contractive Condition
}

\author{
Bhavana Deshrande And AmRish Handa
}

\begin{abstract}
We establish a quadruple coincidence and common quadruple fixed point theorem for hybrid pair of mappings satisfying new contractive condition. It is to be noted that to find quadruple coincidence points, we do not use the condition of continuity of any mapping involved. An example supporting to our result has also been cited. We improve, extend and generalize several known results.
\end{abstract}

\section{Introduction AND PRELIMINARIES}

Let $(X, d)$ be a metric space and $C B(X)$ be the set of all nonempty closed bounded subsets of $X$. Let $D(x, A)$ denote the distance from $x$ to $A \subset X$ and $H$ denote the Hausdorff metric induced by $d$, that is,

$$
\begin{aligned}
D(x, A) & =\inf _{a \in A} d(x, a) \quad \text { and } \\
H(A, B) & =\max \left\{\sup _{a \in A} D(a, B), \sup _{b \in B} D(b, A)\right\}, \quad \text { for all } A, B \in C B(X) .
\end{aligned}
$$

The study of fixed points for multivalued contractions and non-expansive mappings using the Hausdorff metric was initiated by Markin [13]. The existence of fixed points for various multivalued contractive mappings has been studied by many authors under different conditions. The theory of multivalued mappings has applications in control theory, convex optimization, differential inclusion and economics. For details, see $[3,4,10,11,16]$ and the reference therein.

Bhaskar and Lakshmikantham [2] established some coupled fixed point theorems and apply these to study the existence and uniqueness of solution for periodic boundary value problems. Lakshmikantham and Ciric [12] proved coupled coincidence and common coupled fixed point theorems for nonlinear contractive mappings in partially ordered complete metric spaces and extended the results of Bhaskar and Lakshmikantham [2].

2010 Mathematics Subject Classification. Primary: 47H10, 54H25.

Key words and phrases. Quadruple fixed point, quadruple coincidence point, $w$ compatible mappings, F-weakly commuting. 
Karapinar [7] introduced the concept of quadruple fixed point for single valued mappings in partially ordered metric spaces and established some quadruple fixed point theorems.

For more details on quadruple fixed point theory, see $[1,5,6,8,9,14]$. Samet and Vetro [15] introduced the notion of fixed point of $N$ order in case of single-valued mappings. In particular for $N=4$ (quadruple case), we have the following definition:

Definition 1.1. Let $X$ be a non-empty set and $F: X^{4} \rightarrow X$ be a given mapping. An element $(x, y, z, w) \in X^{4}$ is called a quadruple fixed point of the mapping $F$ if

$$
\begin{aligned}
& F(x, y, z, w)=x, F(y, z, w, x)=y, \\
& F(z, w, x, y)=z, F(w, x, y, z)=w .
\end{aligned}
$$

These concepts were extended by Deshpande and Handa [4] to multivalued mappings and obtained quadruple coincidence points and common quadruple fixed point theorems involving hybrid pair of mappings under $\varphi-\psi$ contraction. In [4], Deshpande and Handa introduced the following for multivalued mappings:

Definition 1.2. Let $X$ be a nonempty set, $F: X^{4} \rightarrow 2^{X}$ (a collection of all nonempty subsets of $X$ ) be a multivalued mapping and $g$ be a self-mapping on $X$. An element $(x, y, z, w) \in X^{4}$ is called

(1) A quadruple fixed point of $F$ if $x \in F(x, y, z, w), y \in F(y, z, w, x)$, $z \in F(z, w, x, y)$ and $w \in F(w, x, y, z)$.

(2) A quadruple coincidence point of hybrid pair $\{F, g\}$ if $g(x) \in F(x, y, z, w)$, $g(y) \in F(y, z, w, x), g(z) \in F(z, w, x, y)$ and $g(w) \in F(w, x, y, z)$.

(3) A common quadruple fixed point of hybrid pair $\{F, g\}$ if $x=g(x) \in$ $F(x, y, z, w), y=g(y) \in F(y, z, w, x), z=g(z) \in F(z, w, x, y)$ and $w=g(w) \in F(w, x, y, z)$.

We denote the set of quadruple coincidence points of mappings $F$ and $g$ by $C\{F, g\}$. Note that if $(x, y, z, w) \in C\{F, g\}$, then $(y, z, w, x),(z, w, x, y)$ and $(w, x, y, z)$ are also in $C\{F, g\}$.

Definition 1.3. Let $F: X^{4} \rightarrow 2^{X}$ be a multivalued mapping and $g$ be a self-mapping on $X$. The hybrid pair $\{F, g\}$ is called $w$-compatible if $g(F(x, y, z, w)) \subseteq F(g x, g y, g z, g w)$ whenever $(x, y, z, w) \in C\{F, g\}$.

Definition 1.4. Let $F: X^{4} \rightarrow 2^{X}$ be a multivalued mapping and $g$ be a self-mapping on $X$. The mapping $g$ is called $F$-weakly commuting at some point $(x, y, z, w) \in X^{4}$ if $g^{2} x \in F(g x, g y, g z, g w), g^{2} y \in F(g y, g z, g w, g x)$, $g^{2} z \in F(g z, g w, g x, g y)$ and $g^{2} w \in F(g w, g x, g y, g z)$.

Lemma 1.1. Let $(X, d)$ be a metric space. Then, for each $a \in X$ and $B \in C B(X)$, there is $b_{0} \in B$ such that $D(a, B)=d\left(a, b_{0}\right)$, where $D(a, B)=$ $\inf _{b \in B} d(a, b)$. 
In this paper, we establish a quadruple coincidence and common quadruple fixed point theorem for hybrid pair of mappings satisfying new contractive condition. It is to be noted that to find quadruple coincidence points, we do not use the condition of continuity of any mapping involved. Our result improve, extend, and generalize the results of Bhaskar and Lakshmikantham [2] and Lakshmikantham and Ciric [12]. An example to validate our result has also been demonstrated.

\section{Main Results}

Let $\Phi$ denote the set of all functions $\varphi:[0,+\infty) \rightarrow[0,+\infty)$ satisfying

$\left(i_{\varphi}\right) \varphi$ is non-decreasing,

$\left(i i_{\varphi}\right) \varphi(t)<t$ for all $t>0$,

$\left(i i i_{\varphi}\right) \lim _{r \rightarrow t+} \varphi(r)<t$ for all $t>0$

and $\Psi$ denote the set of all functions $\psi:[0,+\infty) \rightarrow[0,1)$ which satisfies

$\left(i_{\psi}\right) \psi$ is continuous,

$\left(i i_{\psi}\right) \psi(t)<t$ for all $t>0$.

Note that, by $\left(i_{\psi}\right)$ and $\left(i i_{\psi}\right)$ we have that $\psi(t)=0$ if and only if $t=0$.

For simplicity, we define the following:

$$
\begin{aligned}
& M(x, y, z, w, p, q, r, s) \\
& =\min \left\{\begin{array}{c}
D(g x, F(x, y, z, w)), D(g p, F(p, q, r, s)), \\
\ldots, D(g w, F(w, x, y, z)), D(g s, F(s, p, q, r)), \\
D(g x, F(p, q, r, s)), D(g p, F(x, y, z, w)), \\
\ldots, D(g w, F(s, p, q, r)), D(g s, F(w, x, y, z))
\end{array}\right\} \\
& m(x, y, z, w, p, q, r, s) \\
& =\min \left\{\begin{array}{c}
D(x, F(x, y, z, w)), D(p, F(p, q, r, s)), \\
\ldots, D(w, F(w, x, y, z)), D(s, F(s, p, q, r)), \\
D(x, F(p, q, r, s)), D(p, F(x, y, z, w)), \\
\ldots, D(w, F(s, p, q, r)), D(s, F(w, x, y, z))
\end{array}\right\} .
\end{aligned}
$$

Theorem 2.1. Let $(X, d)$ be a metric space. Assume $F: X^{4} \rightarrow C B(X)$ and $g: X \rightarrow X$ be two mappings satisfying

$$
\begin{aligned}
& H(F(x, y, z, w), F(p, q, r, s)) \\
\leq & \varphi\left[\max \left\{\begin{array}{c}
d(g x, g p), d(g y, g q), \\
d(g z, g r), d(g w, g s)
\end{array}\right\}\right]+\psi[M(x, y, z, w, p, q, r, s)],
\end{aligned}
$$

for all $x, y, z, w, p, q, r, s \in X$, where $\varphi \in \Phi$ and $\psi \in \Psi$. Furthermore assume that $F\left(X^{4}\right) \subseteq g(X)$ and $g(X)$ is a complete subset of $X$. Then $F$ and $g$ have 
a quadruple coincidence point. Moreover, $F$ and $g$ have a common quadruple fixed point, if one of the following conditions holds:

(a) F and $g$ are $w$-compatible. $\lim _{n \rightarrow \infty} g^{n} x=p, \lim _{n \rightarrow \infty} g^{n} y=q, \lim _{n \rightarrow \infty} g^{n} z=$ $r$ and $\lim _{n \rightarrow \infty} g^{n} w=s$ for some $(x, y, z, w) \in C\{F, g\}$ and for some $p, q, r, s \in X$ and $g$ is continuous at $p, q, r$ and $s$.

(b) $g$ is $F$-weakly commuting for some $(x, y, z, w) \in C\{F, g\}$ and $g x$, $g y, g z$ and $g w$ are fixed points of $g$, that is, $g^{2} x=g x, g^{2} y=g y$, $g^{2} z=g z$ and $g^{2} w=g w$.

(c) $g$ is continuous at $x, y, z$ and $w$. $\lim _{n \rightarrow \infty} g^{n} p=x, \lim _{n \rightarrow \infty} g^{n} q=y$, $\lim _{n \rightarrow \infty} g^{n} r=z$ and $\lim _{n \rightarrow \infty} g^{n} s=w$ for some $(x, y, z, w) \in C\{F, g\}$ and for some $p, q, r, s \in X$.

(d) $g(C\{F, g\})$ is a singleton subset of $C\{F, g\}$.

Proof. Let $x_{0}, y_{0}, z_{0}, w_{0} \in X$ be arbitrary. Then $F\left(x_{0}, y_{0}, z_{0}, w_{0}\right), F\left(y_{0}, z_{0}\right.$, $\left.w_{0}, x_{0}\right), F\left(z_{0}, w_{0}, x_{0}, y_{0}\right)$ and $F\left(w_{0}, x_{0}, y_{0}, z_{0}\right)$ are well defined. Choose $g x_{1} \in F\left(x_{0}, y_{0}, z_{0}, w_{0}\right), g y_{1} \in F\left(y_{0}, z_{0}, w_{0}, x_{0}\right), g z_{1} \in F\left(z_{0}, w_{0}, x_{0}, y_{0}\right)$ and $g w_{1} \in F\left(w_{0}, x_{0}, y_{0}, z_{0}\right)$, because $F\left(X^{4}\right) \subseteq g(X)$. Since $F: X^{4} \rightarrow C B(X)$, therefore by Lemma 1.1, there exist $u_{1} \in F\left(x_{1}, y_{1}, z_{1}, w_{1}\right), u_{2} \in F\left(y_{1}, z_{1}\right.$, $\left.w_{1}, x_{1}\right), u_{3} \in F\left(z_{1}, w_{1}, x_{1}, y_{1}\right)$ and $u_{4} \in F\left(w_{1}, x_{1}, y_{1}, z_{1}\right)$ such that

$$
\begin{aligned}
d\left(g x_{1}, u_{1}\right) & \leq H\left(F\left(x_{0}, y_{0}, z_{0}, w_{0}\right), F\left(x_{1}, y_{1}, z_{1}, w_{1}\right)\right), \\
d\left(g y_{1}, u_{2}\right) & \leq H\left(F\left(y_{0}, z_{0}, w_{0}, x_{0}\right), F\left(y_{1}, z_{1}, w_{1}, x_{1}\right)\right), \\
d\left(g z_{1}, u_{3}\right) & \leq H\left(F\left(z_{0}, w_{0}, x_{0}, y_{0}\right), F\left(z_{1}, w_{1}, x_{1}, y_{1}\right)\right), \\
d\left(g w_{1}, u_{4}\right) & \leq H\left(F\left(w_{0}, x_{0}, y_{0}, z_{0}\right), F\left(w_{1}, x_{1}, y_{1}, z_{1}\right)\right) .
\end{aligned}
$$

Since $F\left(X^{4}\right) \subseteq g(X)$, there exist $x_{2}, y_{2}, z_{2}, w_{2} \in X$ such that $u_{1}=g x_{2}$, $u_{2}=g y_{2}, u_{3}=g z_{2}$ and $u_{4}=g w_{2}$. Thus

$$
\begin{aligned}
d\left(g x_{1}, g x_{2}\right) & \leq H\left(F\left(x_{0}, y_{0}, z_{0}, w_{0}\right), F\left(x_{1}, y_{1}, z_{1}, w_{1}\right)\right), \\
d\left(g y_{1}, g y_{2}\right) & \leq H\left(F\left(y_{0}, z_{0}, w_{0}, x_{0}\right), F\left(y_{1}, z_{1}, w_{1}, x_{1}\right)\right), \\
d\left(g z_{1}, g z_{2}\right) & \leq H\left(F\left(z_{0}, w_{0}, x_{0}, y_{0}\right), F\left(z_{1}, w_{1}, x_{1}, y_{1}\right)\right), \\
d\left(g w_{1}, g w_{2}\right) & \leq H\left(F\left(w_{0}, x_{0}, y_{0}, z_{0}\right), F\left(w_{1}, x_{1}, y_{1}, z_{1}\right)\right) .
\end{aligned}
$$

Continuing this process, we obtain sequences $\left\{x_{n}\right\},\left\{y_{n}\right\},\left\{z_{n}\right\}$ and $\left\{w_{n}\right\}$ in $X$ such that for all $n \in \mathbb{N}$, we have $g x_{n+1} \in F\left(x_{n}, y_{n}, z_{n}, w_{n}\right), g y_{n+1} \in F\left(y_{n}\right.$, $\left.z_{n}, w_{n}, x_{n}\right), g z_{n+1} \in F\left(z_{n}, w_{n}, x_{n}, y_{n}\right)$ and $g w_{n+1} \in F\left(w_{n}, x_{n}, y_{n}, z_{n}\right)$ such 
that

$$
\begin{aligned}
& d\left(g x_{n}, g x_{n+1}\right) \\
\leq & H\left(F\left(x_{n-1}, y_{n-1}, z_{n-1}, w_{n-1}\right), F\left(x_{n}, y_{n}, z_{n}, w_{n}\right)\right) \\
\leq \varphi & {\left[\max \left\{\begin{array}{c}
d\left(g x_{n-1}, g x_{n}\right), d\left(g y_{n-1}, g y_{n}\right), \\
d\left(g z_{n-1}, g z_{n}\right), d\left(g w_{n-1}, g w_{n}\right)
\end{array}\right\}\right] } \\
& +\psi\left[M\left(x_{n-1}, y_{n-1}, z_{n-1}, w_{n-1}, x_{n}, y_{n}, z_{n}, w_{n}\right)\right] \\
\leq \varphi & {\left[\max \left\{\begin{array}{l}
d\left(g x_{n-1}, g x_{n}\right), d\left(g y_{n-1}, g y_{n}\right), \\
d\left(g z_{n-1}, g z_{n}\right), d\left(g w_{n-1}, g w_{n}\right)
\end{array}\right\}\right] . }
\end{aligned}
$$

Thus

$$
d\left(g x_{n}, g x_{n+1}\right) \leq \varphi\left[\max \left\{\begin{array}{l}
d\left(g x_{n-1}, g x_{n}\right), d\left(g y_{n-1}, g y_{n}\right), \\
d\left(g z_{n-1}, g z_{n}\right), d\left(g w_{n-1}, g w_{n}\right)
\end{array}\right\}\right]
$$

Similarly

$$
\begin{gathered}
d\left(g y_{n}, g y_{n+1}\right) \leq \varphi\left[\max \left\{\begin{array}{l}
d\left(g x_{n-1}, g x_{n}\right), d\left(g y_{n-1}, g y_{n}\right), \\
d\left(g z_{n-1}, g z_{n}\right), d\left(g w_{n-1}, g w_{n}\right)
\end{array}\right\}\right], \\
d\left(g z_{n}, g z_{n+1}\right) \leq \varphi\left[\max \left\{\begin{array}{l}
d\left(g x_{n-1}, g x_{n}\right), d\left(g y_{n-1}, g y_{n}\right), \\
d\left(g z_{n-1}, g z_{n}\right), d\left(g w_{n-1}, g w_{n}\right)
\end{array}\right\}\right], \\
d\left(g w_{n}, g w_{n+1}\right) \leq \varphi\left[\max \left\{\begin{array}{l}
d\left(g x_{n-1}, g x_{n}\right), d\left(g y_{n-1}, g y_{n}\right), \\
d\left(g z_{n-1}, g z_{n}\right), d\left(g w_{n-1}, g w_{n}\right)
\end{array}\right\}\right] .
\end{gathered}
$$

Combining them, we get

$$
\begin{aligned}
& \max \left\{\begin{array}{c}
d\left(g x_{n}, g x_{n+1}\right), d\left(g y_{n}, g y_{n+1}\right), \\
d\left(g z_{n}, g z_{n+1}\right), d\left(g w_{n}, g w_{n+1}\right)
\end{array}\right\} \\
\leq & \varphi\left[\max \left\{\begin{array}{c}
d\left(g x_{n-1}, g x_{n}\right), d\left(g y_{n-1}, g y_{n}\right), \\
d\left(g z_{n-1}, g z_{n}\right), d\left(g w_{n-1}, g w_{n}\right)
\end{array}\right\}\right],
\end{aligned}
$$

which implies, by $\left(i i_{\varphi}\right)$, that

$$
\begin{array}{r}
\max \left\{\begin{array}{l}
d\left(g x_{n}, g x_{n+1}\right), d\left(g y_{n}, g y_{n+1}\right), \\
d\left(g z_{n}, g z_{n+1}\right), d\left(g w_{n}, g w_{n+1}\right)
\end{array}\right\} \\
<\max \left\{\begin{array}{l}
d\left(g x_{n-1}, g x_{n}\right), d\left(g y_{n-1}, g y_{n}\right), \\
d\left(g z_{n-1}, g z_{n}\right), d\left(g w_{n-1}, g w_{n}\right)
\end{array}\right\} .
\end{array}
$$

This shows that the sequence $\left\{\delta_{n}\right\}_{n=0}^{\infty}$ defined by $\delta_{n}=\max \left\{d\left(g x_{n}, g x_{n+1}\right)\right.$, $\left.d\left(g y_{n}, g y_{n+1}\right), d\left(g z_{n}, g z_{n+1}\right), d\left(g w_{n}, g w_{n+1}\right)\right\}$ is a decreasing sequence of positive numbers. Then there exists $\delta \geq 0$ such that

$$
\lim _{n \rightarrow \infty} \delta_{n}=\lim _{n \rightarrow \infty} \max \left\{\begin{array}{l}
d\left(g x_{n}, g x_{n+1}\right), d\left(g y_{n}, g y_{n+1}\right), \\
d\left(g z_{n}, g z_{n+1}\right), d\left(g w_{n}, g w_{n+1}\right)
\end{array}\right\}=\delta .
$$

We shall prove that $\delta=0$. Suppose that $\delta>0$. Letting $n \rightarrow \infty$ in (2), by using (3) and $\left(i i i_{\varphi}\right)$, we get

$$
\delta \leq \lim _{n \rightarrow \infty} \varphi\left(\delta_{n}\right)=\lim _{\delta_{n} \rightarrow \delta+} \varphi\left(\delta_{n}\right)<\delta,
$$


which is a contradiction. Hence

$$
\lim _{n \rightarrow \infty} \delta_{n}=\lim _{n \rightarrow \infty} \max \left\{\begin{array}{l}
d\left(g x_{n}, g x_{n+1}\right), d\left(g y_{n}, g y_{n+1}\right), \\
d\left(g z_{n}, g z_{n+1}\right), d\left(g w_{n}, g w_{n+1}\right)
\end{array}\right\}=0 .
$$

We now prove that $\left\{g x_{n}\right\}_{n=0}^{\infty},\left\{g y_{n}\right\}_{n=0}^{\infty},\left\{g z_{n}\right\}_{n=0}^{\infty}$ and $\left\{g w_{n}\right\}_{n=0}^{\infty}$ are Cauchy sequences in $(X, d)$. Suppose, to the contrary, that one of the sequences is not a Cauchy sequence. Then there exists an $\varepsilon>0$ for which we can find subsequences $\left\{g x_{n(k)}\right\},\left\{g x_{m(k)}\right\}$ of $\left\{g x_{n}\right\}_{n=0}^{\infty},\left\{g y_{n(k)}\right\},\left\{g y_{m(k)}\right\}$ of $\left\{g y_{n}\right\}_{n=0}^{\infty}$, $\left\{g z_{n(k)}\right\},\left\{g z_{m(k)}\right\}$ of $\left\{g z_{n}\right\}_{n=0}^{\infty}$ and $\left\{g w_{n(k)}\right\},\left\{g w_{m(k)}\right\}$ of $\left\{g w_{n}\right\}_{n=0}^{\infty}$ such that

$$
\max \left\{\begin{array}{l}
d\left(g x_{n(k)}, g x_{m(k)}\right), d\left(g y_{n(k)}, g y_{m(k)}\right), \\
d\left(g z_{n(k)}, g z_{m(k)}\right), d\left(g w_{n(k)}, g w_{m(k)}\right)
\end{array}\right\} \geq \varepsilon, \quad k=1,2, \ldots
$$

We can choose $n(k)$ to be the smallest positive integer satisfying (5), so

$$
\max \left\{\begin{array}{l}
d\left(g x_{n(k)-1}, g x_{m(k)}\right), d\left(g y_{n(k)-1}, g y_{m(k)}\right), \\
d\left(g z_{n(k)-1}, g z_{m(k)}\right), d\left(g w_{n(k)-1}, g w_{m(k)}\right)
\end{array}\right\}<\varepsilon .
$$

By (5) and (6) and the triangle inequality, we have

$$
\begin{aligned}
& \varepsilon \leq r_{k}=\max \left\{\begin{array}{c}
d\left(g x_{n(k)}, g x_{m(k)}\right), d\left(g y_{n(k)}, g y_{m(k)}\right), \\
d\left(g z_{n(k)}, g z_{m(k)}\right), d\left(g w_{n(k)}, g w_{m(k)}\right)
\end{array}\right\} \\
& \leq \max \left\{\begin{array}{c}
d\left(g x_{n(k)}, g x_{n(k)-1}\right), d\left(g y_{n(k)}, g y_{n(k)-1}\right), \\
d\left(g z_{n(k)}, g z_{n(k)-1}\right), d\left(g w_{n(k)}, g w_{n(k)-1}\right)
\end{array}\right\} \\
&+\max \left\{\begin{array}{c}
d\left(g x_{n(k)-1}, g x_{m(k)}\right), d\left(g y_{n(k)-1}, g y_{m(k)}\right), \\
d\left(g z_{n(k)-1}, g z_{m(k)}\right), d\left(g w_{n(k)-1}, g w_{m(k)}\right)
\end{array}\right\} \\
&<\max \left\{\begin{array}{l}
d\left(g x_{n(k)}, g x_{n(k)-1}\right), d\left(g y_{n(k)}, g y_{n(k)-1}\right), \\
d\left(g z_{n(k)}, g z_{n(k)-1}\right), d\left(g w_{n(k)}, g w_{n(k)-1}\right)
\end{array}\right\}+\varepsilon .
\end{aligned}
$$

Letting $n \rightarrow \infty$ in the above inequality and using (4), we get

$$
\lim _{k \rightarrow \infty} r_{k}=\lim _{k \rightarrow \infty} \max \left\{\begin{array}{l}
d\left(g x_{n(k)}, g x_{m(k)}\right), d\left(g y_{n(k)}, g y_{m(k)}\right), \\
d\left(g z_{n(k)}, g z_{m(k)}\right), d\left(g w_{n(k)}, g w_{m(k)}\right)
\end{array}\right\}=\varepsilon .
$$

By the triangle inequality, we have

$$
\begin{aligned}
& r_{k}=\max \left\{\begin{array}{l}
d\left(g x_{n(k)}, g x_{m(k)}\right), d\left(g y_{n(k)}, g y_{m(k)}\right), \\
d\left(g z_{n(k)}, g z_{m(k)}\right), d\left(g w_{n(k)}, g w_{m(k)}\right)
\end{array}\right\} \\
& \leq \max \left\{\begin{array}{l}
d\left(g x_{n(k)}, g x_{n(k)+1}\right), d\left(g y_{n(k)}, g y_{n(k)+1}\right), \\
d\left(g z_{n(k)}, g z_{n(k)+1}\right), d\left(g w_{n(k)}, g w_{n(k)+1}\right)
\end{array}\right\} \\
&+ \max \left\{\begin{array}{c}
d\left(g x_{n(k)+1}, g x_{m(k)+1}\right), d\left(g y_{n(k)+1}, g y_{m(k)+1}\right), \\
d\left(g z_{n(k)+1}, g z_{m(k)+1}\right), d\left(g w_{n(k)+1}, g w_{m(k)+1}\right)
\end{array}\right\} \\
&+ \max \left\{\begin{array}{l}
d\left(g x_{m(k)+1}, g x_{m(k)}\right), d\left(g y_{m(k)+1}, g y_{m(k)}\right), \\
d\left(g z_{m(k)+1}, g z_{m(k)}\right), d\left(g w_{m(k)+1}, g w_{m(k)}\right)
\end{array}\right\} \\
& \leq \delta_{n(k)}+\delta_{m(k)}+\max \left\{\begin{array}{l}
d\left(g x_{n(k)+1}, g x_{m(k)+1}\right), d\left(g y_{n(k)+1}, g y_{m(k)+1}\right), \\
d\left(g z_{n(k)+1}, g z_{m(k)+1}\right), d\left(g w_{n(k)+1}, g w_{m(k)+1}\right)
\end{array}\right\} .
\end{aligned}
$$


Thus

$$
r_{k} \leq \delta_{n(k)}+\delta_{m(k)}
$$

$$
+\max \left\{\begin{array}{c}
d\left(g x_{n(k)+1}, g x_{m(k)+1}\right), d\left(g y_{n(k)+1}, g y_{m(k)+1}\right), \\
d\left(g z_{n(k)+1}, g z_{m(k)+1}\right), d\left(g w_{n(k)+1}, g w_{m(k)+1}\right)
\end{array}\right\} .
$$

Since

$$
\begin{aligned}
g x_{n(k)+1} & \in F\left(x_{n(k)}, y_{n(k)}, z_{n(k)}, w_{n(k)}\right), \\
g x_{m(k)+1} & \in F\left(x_{m(k)}, y_{m(k)}, z_{m(k)}, w_{m(k)}\right), \\
g y_{n(k)+1} & \in F\left(y_{n(k)}, z_{n(k)}, w_{n(k)}, x_{n(k)}\right), \\
g y_{m(k)+1} & \in F\left(y_{m(k)}, z_{m(k)}, w_{m(k)}, x_{m(k)}\right), \\
g z_{n(k)+1} & \in F\left(z_{n(k)}, w_{n(k)}, x_{n(k)}, y_{n(k)}\right), \\
g z_{m(k)+1} & \in F\left(z_{m(k)}, w_{m(k)}, x_{m(k)}, y_{m(k)}\right), \\
g w_{n(k)+1} & \in F\left(w_{n(k)}, x_{n(k)}, y_{n(k)}, z_{n(k)}\right) \text { and } \\
g w_{m(k)+1} & \in F\left(w_{m(k)}, x_{m(k)}, y_{m(k)}, z_{m(k)}\right),
\end{aligned}
$$

therefore by (1) and triangle inequality, we have

$$
\begin{aligned}
& d\left(g x_{n(k)+1}, g x_{m(k)+1}\right) \\
\leq & H\left(F\left(x_{n(k)}, y_{n(k)}, z_{n(k)}, w_{n(k)}\right), F\left(x_{m(k)}, y_{m(k)}, z_{m(k)}, w_{m(k)}\right)\right) \\
\leq & {\left[\max \left\{\begin{array}{c}
d\left(g x_{n(k)}, g x_{m(k)}\right), d\left(g y_{n(k)}, g y_{m(k)}\right), \\
d\left(g z_{n(k)}, g z_{m(k)}\right), d\left(g w_{n(k)}, g w_{m(k)}\right)
\end{array}\right\}\right] } \\
& +\psi\left[M\left(\begin{array}{c}
x_{n(k)}, y_{n(k)}, z_{n(k)}, w_{n(k)}, \\
x_{m(k)}, y_{m(k)}, z_{m(k)}, w_{m(k)}
\end{array}\right)\right] \\
\leq & \varphi\left(r_{k}\right)+\psi\left[M\left(\begin{array}{c}
x_{n(k)}, y_{n(k)}, z_{n(k)}, w_{n(k)}, \\
x_{m(k)}, y_{m(k)}, z_{m(k)}, w_{m(k)}
\end{array}\right)\right] .
\end{aligned}
$$

Thus

$$
d\left(g x_{n(k)+1}, g x_{m(k)+1}\right) \leq \varphi\left(r_{k}\right)+\psi\left[M\left(\begin{array}{c}
x_{n(k)}, y_{n(k)}, z_{n(k)}, w_{n(k)}, \\
x_{m(k)}, y_{m(k)}, z_{m(k)}, w_{m(k)}
\end{array}\right)\right]
$$

Similarly

$$
\begin{gathered}
d\left(g y_{n(k)+1}, g y_{m(k)+1}\right) \leq \varphi\left(r_{k}\right)+\psi\left[M\left(\begin{array}{c}
x_{n(k)}, y_{n(k)}, z_{n(k)}, w_{n(k)}, \\
x_{m(k)}, y_{m(k)}, z_{m(k)}, w_{m(k)}
\end{array}\right)\right], \\
d\left(g z_{n(k)+1}, g z_{m(k)+1}\right) \leq \varphi\left(r_{k}\right)+\psi\left[M\left(\begin{array}{c}
x_{n(k)}, y_{n(k)}, z_{n(k)}, w_{n(k)}, \\
x_{m(k)}, y_{m(k)}, z_{m(k)}, w_{m(k)}
\end{array}\right)\right], \\
d\left(g w_{n(k)+1}, g w_{m(k)+1}\right) \leq \varphi\left(r_{k}\right)+\psi\left[M\left(\begin{array}{c}
x_{n(k)}, y_{n(k)}, z_{n(k)}, w_{n(k)}, \\
x_{m(k)}, y_{m(k)}, z_{m(k)}, w_{m(k)}
\end{array}\right)\right] .
\end{gathered}
$$


Combining them, we get

$$
\begin{aligned}
& \max \left\{\begin{array}{r}
d\left(g x_{n(k)+1}, g x_{m(k)+1}\right), d\left(g y_{n(k)+1}, g y_{m(k)+1}\right), \\
d\left(g z_{n(k)+1}, g z_{m(k)+1}\right), d\left(g w_{n(k)+1}, g w_{m(k)+1}\right)
\end{array}\right\} \\
\leq & \varphi\left(r_{k}\right)+\psi\left[M\left(\begin{array}{c}
x_{n(k)}, y_{n(k)}, z_{n(k)}, w_{n(k)}, \\
x_{m(k)}, y_{m(k)}, z_{m(k)}, w_{m(k)}
\end{array}\right)\right] .
\end{aligned}
$$

By (8) and (9), we get

$$
r_{k} \leq \delta_{n(k)}+\delta_{m(k)}+\varphi\left(r_{k}\right)+\psi\left[M\left(\begin{array}{c}
x_{n(k)}, y_{n(k)}, z_{n(k)}, w_{n(k)}, \\
x_{m(k)}, y_{m(k)}, z_{m(k)}, w_{m(k)}
\end{array}\right)\right] .
$$

Letting $k \rightarrow \infty$ in the above inequality, by using (4), (7), (A), $\left(i_{\psi}\right),\left(i i_{\psi}\right)$ and $\left(i i i_{\varphi}\right)$, we get

$$
\varepsilon \leq 0+0+\lim _{k \rightarrow \infty} \varphi\left(r_{k}\right)+0 \leq \lim _{r_{k} \rightarrow \varepsilon+} \varphi\left(r_{k}\right)<\varepsilon,
$$

which is a contradiction. This shows that $\left\{g x_{n}\right\}_{n=0}^{\infty},\left\{g y_{n}\right\}_{n=0}^{\infty},\left\{g z_{n}\right\}_{n=0}^{\infty}$ and $\left\{g w_{n}\right\}_{n=0}^{\infty}$ are Cauchy sequences in $g(X)$. Since $g(X)$ is complete, therefore there exist $x, y, z, w \in X$ such that

$$
\begin{array}{lll}
\lim _{n \rightarrow \infty} g x_{n}=g x, & \lim _{n \rightarrow \infty} g y_{n}=g y, \\
\lim _{n \rightarrow \infty} g z_{n}=g z & \text { and } & \lim _{n \rightarrow \infty} g w_{n}=g w .
\end{array}
$$

Now, since $g x_{n+1} \in F\left(x_{n}, y_{n}, z_{n}, w_{n}\right), g y_{n+1} \in F\left(y_{n}, z_{n}, w_{n}, x_{n}\right), g z_{n+1} \in$ $F\left(z_{n}, w_{n}, x_{n}, y_{n}\right)$ and $g w_{n+1} \in F\left(w_{n}, x_{n}, y_{n}, z_{n}\right)$, therefore by using condition (1), we get

$$
\begin{aligned}
& D\left(g x_{n+1}, F(x, y, z, w)\right) \\
\leq & H\left(F\left(x_{n}, y_{n}, z_{n}, w_{n}\right), F(x, y, z, w)\right) \\
\leq & \varphi\left[\max \left\{d\left(g x_{n}, g x\right), d\left(g y_{n}, g y\right), d\left(g z_{n}, g z\right), d\left(g w_{n}, g w\right)\right\}\right] \\
& +\psi\left[M\left(x_{n}, y_{n}, z_{n}, w_{n}, x, y, z, w\right)\right] .
\end{aligned}
$$

Letting $n \rightarrow \infty$ in the above inequality, by using (10), (A), $\left(i_{\psi}\right),\left(i i_{\psi}\right)$ and $\left(i i i_{\varphi}\right)$, we get

$$
D(g x, F(x, y, z, w)) \leq \lim _{t \rightarrow 0+} \varphi(t)+0=0+0=0,
$$

which implies that

$$
D(g x, F(x, y, z, w))=0 .
$$

Similarly, we can get

$$
\begin{aligned}
& D(g y, F(y, z, w, x))=0, \\
& D(g z, F(z, w, x, y))=0, \\
& D(g w, F(w, x, y, z))=0,
\end{aligned}
$$


it follows that

$$
\begin{aligned}
& g x \in F(x, y, z, w), g y \in F(y, z, w, x), \\
& g z \in F(z, w, x, y), g w \in F(w, x, y, z),
\end{aligned}
$$

that is, $(x, y, z, w)$ is a quadruple coincidence point of $F$ and $g$.

Suppose now that (a) holds. Assume that for some $(x, y, z, w) \in C\{F, g\}$

$$
\lim _{n \rightarrow \infty} g^{n} x=p, \quad \lim _{n \rightarrow \infty} g^{n} y=q, \quad \lim _{n \rightarrow \infty} g^{n} z=r \text { and } \lim _{n \rightarrow \infty} g^{n} w=s,
$$

where $p, q, r, s \in X$. Since $g$ is continuous at $p, q, r$ and $s$, we have, by (11), that $p, q, r$ and $s$ are fixed points of $g$, that is,

$$
g p=p, \quad g q=q, \quad g r=r \quad \text { and } \quad g s=s .
$$

As $F$ and $g$ are $w$-compatible, so for all $n \geq 1$

$$
\begin{aligned}
g^{n} x & \in F\left(g^{n-1} x, g^{n-1} y, g^{n-1} z, g^{n-1} w\right), \\
g^{n} y & \in F\left(g^{n-1} y, g^{n-1} z, g^{n-1} w, g^{n-1} x\right), \\
g^{n} z & \in F\left(g^{n-1} z, g^{n-1} w, g^{n-1} x, g^{n-1} y\right), \\
g^{n} w & \in F\left(g^{n-1} w, g^{n-1} x, g^{n-1} y, g^{n-1} z\right) .
\end{aligned}
$$

By using (1) and (13), we obtain

$$
\begin{aligned}
& D\left(g^{n} x, F(p, q, r, s)\right) \\
\leq & H\left(F\left(g^{n-1} x, g^{n-1} y, g^{n-1} z, g^{n-1} w\right), F(p, q, r, s)\right) \\
\leq & \varphi\left[\max \left\{d\left(g^{n} x, g p\right), d\left(g^{n} y, g q\right), d\left(g^{n} z, g r\right), d\left(g^{n} w, g s\right)\right\}\right] \\
& +\psi\left[M\left(g^{n-1} x, g^{n-1} y, g^{n-1} z, g^{n-1} w, p, q, r, s\right)\right] .
\end{aligned}
$$

On taking limit as $n \rightarrow \infty$ in the above inequality, by using (11), (12), (A), $\left(i_{\psi}\right),\left(i i_{\psi}\right)$ and $\left(i i i_{\varphi}\right)$, we get

$$
D(g p, F(p, q, r, s)) \leq \lim _{t \rightarrow 0+} \varphi(t)+0=0+0=0
$$

which implies that

$$
D(g p, F(p, q, r, s))=0
$$

Similarly we can get

$$
D(g q, F(q, r, s, p))=D(g r, F(r, s, p, q))=D(g s, F(s, p, q, r))=0,
$$

which implies that

$$
\begin{aligned}
& g p \in F(p, q, r, s), \quad g q \in F(q, r, s, p), \\
& g r \in F(r, s, p, q), \quad g s \in F(s, p, q, r) .
\end{aligned}
$$


By (12) and (14), we get

$$
\begin{aligned}
& p=g p \in F(p, q, r, s), \\
& q=g q \in F(q, r, s, p), \\
& r=g r \in F(r, s, p, q), \\
& s=g s \in F(s, p, q, r),
\end{aligned}
$$

that is, $(p, q, r, s)$ is a common quadruple fixed point of $F$ and $g$.

Suppose now that $(b)$ holds. Assume that for some $(x, y, z, w) \in C\{F, g\}$, $g$ is $F$-weakly commuting, that is,

$$
\begin{array}{ll}
g^{2} x \in F(g x, g y, g z, g w), & g^{2} y \in F(g y, g z, g w, g x), \\
g^{2} z \in F(g z, g w, g x, g y), & g^{2} w \in F(g w, g x, g y, g z)
\end{array}
$$

and

Thus

$$
g^{2} x=g x, \quad g^{2} y=g y, \quad g^{2} z=g z, \quad g^{2} w=g w .
$$

$$
\begin{aligned}
& g x=g^{2} x \in F(g x, g y, g z, g w), \quad g y=g^{2} y \in F(g y, g z, g w, g x), \\
& g z=g^{2} z \in F(g z, g w, g x, g y), \quad g w=g^{2} w \in F(g w, g x, g y, g z),
\end{aligned}
$$

that is, $(g x, g y, g z, g w)$ is a common quadruple fixed point of $F$ and $g$.

Suppose now that $(c)$ holds. Assume that for some $(x, y, z, w) \in C\{F, g\}$ and for some $p, q, r, s \in X, \lim _{n \rightarrow \infty} g^{n} p=x, \lim _{n \rightarrow \infty} g^{n} q=y, \lim _{n \rightarrow \infty} g^{n} r=z$ and $\lim _{n \rightarrow \infty} g^{n} s=w$. Since $g$ is continuous at $x, y, z$ and $w$, therefore $x, y, z$ and $w$ are fixed points of $g$, that is, $g x=x, g y=y, g z=z$ and $g w=w$. Since $(x, y, z, w) \in C\{F, g\}$. Therefore, we get $x=g x \in F(x, y, z, w)$, $y=g y \in F(y, z, w, x), z=g z \in F(z, w, x, y), w=g w \in F(w, x, y, z)$, that is, $(x, y, z, w)$ is a common quadruple fixed point of $F$ and $g$.

Finally, suppose that $(d)$ holds. Let $g(C\{F, g\})=\{(x, x, x, x)\}$. Then $\{x\}=\{g x\}=F(x, x, x, x)$. Hence $(x, x, x, x)$ is quadruple fixed point of $F$ and $g$.

Example 2.1. Suppose that $X=[0,1]$, equipped with the metric $d: X \times$ $X \rightarrow[0,+\infty)$ defined by $d(x, y)=\max \{x, y\}$ and $d(x, x)=0$ for all $x, y \in$ $X$. Let $F: X \times X \times X \rightarrow C B(X)$ be defined as

$$
F(x, y, z, w)=\left\{\begin{array}{c}
\{0\}, \text { for } x, y, z, w=1 \\
{\left[0, \frac{x^{2}+y^{2}+z^{2}+w^{2}}{8}\right], \text { for } x, y, z, w \in[0,1)}
\end{array}\right.
$$

and $g: X \rightarrow X$ be defined as

$$
g(x)=x^{2}, \text { for all } x \in X .
$$

Define $\varphi:[0,+\infty) \rightarrow[0,+\infty)$ by

$$
\varphi(t)=\left\{\begin{array}{l}
\frac{t}{2}, \text { for } t \neq 1 \\
\frac{3}{4}, \text { for } t=1
\end{array}\right.
$$


and $\psi:[0,+\infty) \rightarrow[0,1)$ by

$$
\psi(t)=\frac{t}{4}, \text { for } t \geq 0 .
$$

Now, for all $x, y, z, w, p, q, r, s \in X$ with $x, y, z, w, p, q, r, s \in[0,1)$, we have Case (a). If $x^{2}+y^{2}+z^{2}+w^{2}=p^{2}+q^{2}+r^{2}+s^{2}$, then

$$
\begin{aligned}
& H(F(x, y, z, w), F(p, q, r, s)) \\
\leq & \frac{p^{2}+q^{2}+r^{2}+s^{2}}{8} \\
\leq & \frac{1}{8} \max \left\{x^{2}, p^{2}\right\}+\frac{1}{8} \max \left\{y^{2}, q^{2}\right\}+\frac{1}{8} \max \left\{z^{2}, r^{2}\right\}+\frac{1}{8} \max \left\{w^{2}, s^{2}\right\} \\
\leq & \frac{1}{8} d(g x, g p)+\frac{1}{8} d(g y, g q)+\frac{1}{8} d(g z, g r)+\frac{1}{8} d(g w, g s) \\
\leq & \frac{1}{8} \max \{d(g x, g p), d(g y, g q), d(g z, g r), d(g w, g s)\} \\
& +\frac{1}{8} \max \{d(g x, g p), d(g y, g q), d(g z, g r), d(g w, g s)\} \\
& +\frac{1}{8} \max \{d(g x, g p), d(g y, g q), d(g z, g r), d(g w, g s)\} \\
& +\frac{1}{8} \max \{d(g x, g p), d(g y, g q), d(g z, g r), d(g w, g s)\} \\
\leq & \frac{1}{2} \max \{d(g x, g p), d(g y, g q), d(g z, g r), d(g w, g s)\} \\
\leq & \varphi \max \{d(g x, g p), d(g y, g q), d(g z, g r), d(g w, g s)\}] \\
& +\psi[M(x, y, z, w, p, q, r, s)] .
\end{aligned}
$$

Case (b). If $x^{2}+y^{2}+z^{2}+w^{2} \neq p^{2}+q^{2}+r^{2}+s^{2}$ with $x^{2}+y^{2}+z^{2}+w^{2}<$ $p^{2}+q^{2}+r^{2}+s^{2}$, then

$$
\begin{aligned}
& H(F(x, y, z, w), F(p, q, r, s)) \\
= & \frac{p^{2}+q^{2}+r^{2}+s^{2}}{8} \\
\leq & \frac{1}{8} \max \left\{x^{2}, p^{2}\right\}+\frac{1}{8} \max \left\{y^{2}, q^{2}\right\}+\frac{1}{8} \max \left\{z^{2}, r^{2}\right\}+\frac{1}{8} \max \left\{w^{2}, s^{2}\right\} \\
\leq & \frac{1}{8} d(g x, g p)+\frac{1}{8} d(g y, g q)+\frac{1}{8} d(g z, g r)+\frac{1}{8} d(g w, g s) \\
\leq & \frac{1}{8} \max \{d(g x, g p), d(g y, g q), d(g z, g r), d(g w, g s)\}
\end{aligned}
$$




$$
\begin{aligned}
&+ \frac{1}{8} \max \{d(g x, g p), d(g y, g q), d(g z, g r), d(g w, g s)\} \\
&+ \frac{1}{8} \max \{d(g x, g p), d(g y, g q), d(g z, g r), d(g w, g s)\} \\
&+\frac{1}{8} \max \{d(g x, g p), d(g y, g q), d(g z, g r), d(g w, g s)\} \\
& \leq \frac{1}{2}[\max \{d(g x, g p), d(g y, g q), d(g z, g r), d(g w, g s)\}] \\
& \leq \varphi[\max \{d(g x, g p), d(g y, g q), d(g z, g r), d(g w, g s)\}] \\
& \quad+\psi[M(x, y, z, w, p, q, r, s)] .
\end{aligned}
$$

Similarly, we obtain the same result for $p^{2}+q^{2}+r^{2}+s^{2}<x^{2}+y^{2}+z^{2}+w^{2}$. Thus the contractive condition (1) is satisfied for all $x, y, z, w, p, q, r, s \in X$ with $x, y, z, w, p, q, r, s \in[0,1)$. Again, for all $x, y, z, w, p, q, r, s \in X$ with $x, y, z, w \in[0,1)$ and $p, q, r, s=1$, we have

$$
\begin{aligned}
& H(F(x, y, z, w), F(p, q, r, s)) \\
= & \frac{x^{2}+y^{2}+z^{2}+w^{2}}{8} \\
\leq & \frac{1}{8} \max \left\{x^{2}, p^{2}\right\}+\frac{1}{8} \max \left\{y^{2}, q^{2}\right\}+\frac{1}{8} \max \left\{z^{2}, r^{2}\right\}+\frac{1}{8} \max \left\{w^{2}, s^{2}\right\} \\
\leq & \frac{1}{8} d(g x, g p)+\frac{1}{8} d(g y, g q)+\frac{1}{8} d(g z, g r)+\frac{1}{8} d(g w, g s) \\
\leq & \frac{1}{8} \max \{d(g x, g p), d(g y, g q), d(g z, g r), d(g w, g s)\} \\
& +\frac{1}{8} \max \{d(g x, g p), d(g y, g q), d(g z, g r), d(g w, g s)\} \\
& +\frac{1}{8} \max \{d(g x, g p), d(g y, g q), d(g z, g r), d(g w, g s)\} \\
& \quad+\frac{1}{8} \max \{d(g x, g p), d(g y, g q), d(g z, g r), d(g w, g s)\} \\
\leq & \frac{1}{2}[\max \{d(g x, g p), d(g y, g q), d(g z, g r), d(g w, g s)\}] \\
\leq & \varphi[\max \{d(g x, g p), d(g y, g q), d(g z, g r), d(g w, g s)\}] \\
& +\psi \psi
\end{aligned}
$$

Thus the contractive condition (1) is satisfied for all $x, y, z, w, p, q, r, s \in X$ with $x, y, z, w \in[0,1)$ and $p, q, r, s=1$. Similarly, we can see that the contractive condition (1) is satisfied for all $x, y, z, w, p, q, r, s \in X$ with $x, y, z, w, p, q, r, s=1$. Hence, the hybrid pair $\{F, g\}$ satisfies the contractive condition (1), for all $x, y, z, w, p, q, r, s \in X$. In addition, all the other conditions of Theorem 2.1 are satisfied and $z=(0,0,0,0)$ is a common quadruple 
fixed point of hybrid pair $\{F, g\}$. The function $F: X^{4} \rightarrow C B(X)$ involved in this example is not continuous on $X^{4}$.

Corollary 2.1. Let $(X, d)$ be a metric space. Assume $F: X^{4} \rightarrow C B(X)$ and $g: X \rightarrow X$ be two mappings satisfying

$$
\begin{aligned}
& H(F(x, y, z, w), F(p, q, r, s)) \\
\leq \varphi & {\left[\frac{d(g x, g p)+d(g y, g q)+d(g z, g r)+d(g w, g s)}{4}\right] } \\
& +\psi[M(x, y, z, w, p, q, r, s)],
\end{aligned}
$$

for all $x, y, z, w, p, q, r, s \in X$, where $\varphi \in \Phi$ and $\psi \in \Psi$. Furthermore assume that $F\left(X^{4}\right) \subseteq g(X)$ and $g(X)$ is a complete subset of $X$. Then $F$ and $g$ have a quadruple coincidence point. Moreover, $F$ and $g$ have a common quadruple fixed point, if one of the following conditions holds:

(a) $F$ and $g$ are $w$-compatible. $\lim _{n \rightarrow \infty} g^{n} x=p, \lim _{n \rightarrow \infty} g^{n} y=q, \lim _{n \rightarrow \infty} g^{n} z=$ $r$ and $\lim _{n \rightarrow \infty} g^{n} w=s$ for some $(x, y, z, w) \in C\{F, g\}$ and for some $p, q, r, s \in X$ and $g$ is continuous at $p, q, r$ and $s$.

(b) $g$ is $F$-weakly commuting for some $(x, y, z, w) \in C\{F, g\}$ and $g x$, $g y, g z$ and $g w$ are fixed points of $g$, that is, $g^{2} x=g x, g^{2} y=g y$, $g^{2} z=g z$ and $g^{2} w=g w$.

(c) $g$ is continuous at $x, y, z$ and $w$. $\lim _{n \rightarrow \infty} g^{n} p=x, \lim _{n \rightarrow \infty} g^{n} q=y$, $\lim _{n \rightarrow \infty} g^{n} r=z$ and $\lim _{n \rightarrow \infty} g^{n} s=w$ for some $(x, y, z, w) \in C\{F, g\}$ and for some $p, q, r, s \in X$.

(d) $g(C\{F, g\})$ is a singleton subset of $C\{F, g\}$.

Proof. It suffices to remark that

$$
\begin{aligned}
& \frac{d(g x, g p)+d(g y, g q)+d(g z, g r)+d(g w, g s)}{4} \\
\leq & \frac{\max \{d(g x, g p), d(g y, g q), d(g z, g r), d(g w, g s)\}}{4} \\
& +\frac{\max \{d(g x, g p), d(g y, g q), d(g z, g r), d(g w, g s)\}}{4} \\
& +\frac{\max \{d(g x, g p), d(g y, g q), d(g z, g r), d(g w, g s)\}}{4} \\
& +\frac{\max \{d(g x, g p), d(g y, g q), d(g z, g r), d(g w, g s)\}}{4} \\
\leq & \max \{d(g x, g p), d(g y, g q), d(g z, g r), d(g w, g s)\} .
\end{aligned}
$$

Then, we apply Theorem 2.1 , since $\varphi$ is non-decreasing.

If we put $g=I$ (the identity mapping) in the Theorem 2.1, we get the following result: 
Corollary 2.2. Let $(X, d)$ be a complete metric space, $F: X^{4} \rightarrow C B(X)$ be a mapping satisfying

$$
\begin{aligned}
H & (F(x, y, z, w), F(p, q, r, s)) \\
\leq \varphi & {[\max \{d(x, p), d(y, q), d(z, r), d(w, s)\}] } \\
& +\psi[m(x, y, z, w, p, q, r, s)],
\end{aligned}
$$

for all $x, y, z, w, p, q, r, s \in X$, where $\varphi \in \Phi$ and $\psi \in \Psi$. Then $F$ has a quadruple fixed point.

If we put $g=I$ (the identity mapping) in the Corollary 2.1, we get the following result:

Corollary 2.3. Let $(X, d)$ be a complete metric space, $F: X^{4} \rightarrow C B(X)$ be a mapping satisfying

$$
\begin{aligned}
& H(F(x, y, z, w), F(p, q, r, s)) \\
\leq \varphi & {\left[\frac{d(x, p)+d(y, q)+d(z, r)+d(w, s)}{4}\right] } \\
& +\psi[m(x, y, z, w, p, q, r, s)],
\end{aligned}
$$

for all $x, y, z, w, p, q, r, s \in X$, where $\varphi \in \Phi$ and $\psi \in \Psi$. Then $F$ has a quadruple fixed point.

If we put $\psi(t)=0$ in Theorem 2.1, we get the following result:

Corollary 2.4. Let $(X, d)$ be a metric space. Assume $F: X^{4} \rightarrow C B(X)$ and $g: X \rightarrow X$ be two mappings satisfying

$$
H(F(x, y, z, w), F(p, q, r, s)) \leq \varphi\left[\max \left\{\begin{array}{c}
d(g x, g p), d(g y, g q), \\
d(g z, g r), d(g w, g s)
\end{array}\right\}\right],
$$

for all $x, y, z, w, p, q, r, s \in X$, where $\varphi \in \Phi$. Furthermore assume that $F\left(X^{4}\right) \subseteq g(X)$ and $g(X)$ is a complete subset of $X$. Then $F$ and $g$ have $a$ quadruple coincidence point. Moreover, $F$ and $g$ have a common quadruple fixed point, if one of the following conditions holds:

(a) $F$ and $g$ are $w$-compatible. $\lim _{n \rightarrow \infty} g^{n} x=p, \lim _{n \rightarrow \infty} g^{n} y=q, \lim _{n \rightarrow \infty} g^{n} z=$ $r$ and $\lim _{n \rightarrow \infty} g^{n} w=s$ for some $(x, y, z, w) \in C\{F, g\}$ and for some $p, q, r, s \in X$ and $g$ is continuous at $p, q, r$ and $s$.

(b) $g$ is $F$-weakly commuting for some $(x, y, z, w) \in C\{F, g\}$ and $g x$, $g y, g z$ and $g w$ are fixed points of $g$, that is, $g^{2} x=g x, g^{2} y=g y$, $g^{2} z=g z$ and $g^{2} w=g w$.

(c) $g$ is continuous at $x, y, z$ and $w . \lim _{n \rightarrow \infty} g^{n} p=x, \lim _{n \rightarrow \infty} g^{n} q=y$, $\lim _{n \rightarrow \infty} g^{n} r=z$ and $\lim _{n \rightarrow \infty} g^{n} s=w$ for some $(x, y, z, w) \in C\{F, g\}$ and for some $p, q, r, s \in X$.

(d) $g(C\{F, g\})$ is a singleton subset of $C\{F, g\}$.

If we put $\psi(t)=0$ in Corollary 2.1, we get the following result: 
Corollary 2.5. Let $(X, d)$ be a metric space. Assume $F: X^{4} \rightarrow C B(X)$ and $g: X \rightarrow X$ be two mappings satisfying

$$
\begin{aligned}
& H(F(x, y, z, w), F(p, q, r, s)) \\
\leq & \varphi\left[\frac{d(g x, g p)+d(g y, g q)+d(g z, g r)+d(g w, g s)}{4}\right],
\end{aligned}
$$

for all $x, y, z, w, p, q, r, s \in X$, where $\varphi \in \Phi$. Furthermore assume that $F\left(X^{4}\right) \subseteq g(X)$ and $g(X)$ is a complete subset of $X$. Then $F$ and $g$ have $a$ quadruple coincidence point. Moreover, $F$ and $g$ have a common quadruple fixed point, if one of the following conditions holds:

(a) F and $g$ are $w$-compatible. $\lim _{n \rightarrow \infty} g^{n} x=p, \lim _{n \rightarrow \infty} g^{n} y=q, \lim _{n \rightarrow \infty} g^{n} z=$ $r$ and $\lim _{n \rightarrow \infty} g^{n} w=s$ for some $(x, y, z, w) \in C\{F, g\}$ and for some $p, q, r, s \in X$ and $g$ is continuous at $p, q, r$ and $s$.

(b) $g$ is $F$-weakly commuting for some $(x, y, z, w) \in C\{F, g\}$ and $g x$, $g y, g z$ and $g w$ are fixed points of $g$, that is, $g^{2} x=g x, g^{2} y=g y$, $g^{2} z=g z$ and $g^{2} w=g w$.

(c) $g$ is continuous at $x, y, z$ and $w . \lim _{n \rightarrow \infty} g^{n} p=x, \lim _{n \rightarrow \infty} g^{n} q=y$, $\lim _{n \rightarrow \infty} g^{n} r=z$ and $\lim _{n \rightarrow \infty} g^{n} s=w$ for some $(x, y, z, w) \in C\{F, g\}$ and for some $p, q, r, s \in X$.

(d) $g(C\{F, g\})$ is a singleton subset of $C\{F, g\}$.

If we put $g=I$ (the identity mapping) in the Corollary 2.4, we get the following result:

Corollary 2.6. Let $(X, d)$ be a complete metric space, $F: X^{4} \rightarrow C B(X)$ be a mapping satisfying

$$
H(F(x, y, z, w), F(p, q, r, s)) \leq \varphi\left[\max \left\{\begin{array}{c}
d(x, p), d(y, q), \\
d(z, r), d(w, s)
\end{array}\right\}\right],
$$

for all $x, y, z, w, p, q, r, s \in X$, where $\varphi \in \Phi$. Then $F$ has a quadruple fixed point.

If we put $g=I$ (the identity mapping) in the Corollary 2.5, we get the following result:

Corollary 2.7. Let $(X, d)$ be a complete metric space, $F: X^{4} \rightarrow C B(X)$ be a mapping satisfying

$$
H(F(x, y, z, w), F(p, q, r, s)) \leq \varphi\left[\frac{d(x, p)+d(y, q)+d(z, r)+d(w, s)}{4}\right],
$$

for all $x, y, z, w, p, q, r, s \in X$, where $\varphi \in \Phi$. Then $F$ has a quadruple fixed point.

If we put $\varphi(t)=k t$ where $0<k<1$ in Corollary 2.4, we get the following result: 
Corollary 2.8. Let $(X, d)$ be a metric space. Assume $F: X^{4} \rightarrow C B(X)$ and $g: X \rightarrow X$ be two mappings satisfying

$$
H(F(x, y, z, w), F(p, q, r, s)) \leq k \max \left\{\begin{array}{c}
d(g x, g p), d(g y, g q), \\
d(g z, g r), d(g w, g s)
\end{array}\right\},
$$

for all $x, y, z, w, p, q, r, s \in X$, where $0<k<1$. Furthermore assume that $F\left(X^{4}\right) \subseteq g(X)$ and $g(X)$ is a complete subset of $X$. Then $F$ and $g$ have $a$ quadruple coincidence point. Moreover, $F$ and $g$ have a common quadruple fixed point, if one of the following conditions holds:

(a) $F$ and $g$ are $w$-compatible. $\lim _{n \rightarrow \infty} g^{n} x=p, \lim _{n \rightarrow \infty} g^{n} y=q, \lim _{n \rightarrow \infty} g^{n} z=$ $r$ and $\lim _{n \rightarrow \infty} g^{n} w=s$ for some $(x, y, z, w) \in C\{F, g\}$ and for some $p, q, r, s \in X$ and $g$ is continuous at $p, q, r$ and $s$.

(b) $g$ is F-weakly commuting for some $(x, y, z, w) \in C\{F, g\}$ and $g x$, $g y, g z$ and $g w$ are fixed points of $g$, that is, $g^{2} x=g x, g^{2} y=g y$, $g^{2} z=g z$ and $g^{2} w=g w$.

(c) $g$ is continuous at $x, y, z$ and $w . \lim _{n \rightarrow \infty} g^{n} p=x, \lim _{n \rightarrow \infty} g^{n} q=y$, $\lim _{n \rightarrow \infty} g^{n} r=z$ and $\lim _{n \rightarrow \infty} g^{n} s=w$ for some $(x, y, z, w) \in C\{F, g\}$ and for some $p, q, r, s \in X$.

(d) $g(C\{F, g\})$ is a singleton subset of $C\{F, g\}$.

If we put $\varphi(t)=k t$ where $0<k<1$ in Corollary 2.5, we get the following result:

Corollary 2.9. Let $(X, d)$ be a metric space. Assume $F: X^{4} \rightarrow C B(X)$ and $g: X \rightarrow X$ be two mappings satisfying

$$
\begin{aligned}
& H(F(x, y, z, w), F(p, q, r, s)) \\
\leq & k\left[\frac{d(g x, g p)+d(g y, g q)+d(g z, g r)+d(g w, g s)}{4}\right],
\end{aligned}
$$

for all $x, y, z, w, p, q, r, s \in X$, where $0<k<1$. Furthermore assume that $F\left(X^{4}\right) \subseteq g(X)$ and $g(X)$ is a complete subset of $X$. Then $F$ and $g$ have a quadruple coincidence point. Moreover, $F$ and $g$ have a common quadruple fixed point, if one of the following conditions holds:

(a) F and $g$ are $w$-compatible. $\lim _{n \rightarrow \infty} g^{n} x=p, \lim _{n \rightarrow \infty} g^{n} y=q, \lim _{n \rightarrow \infty} g^{n} z=$ $r$ and $\lim _{n \rightarrow \infty} g^{n} w=s$ for some $(x, y, z, w) \in C\{F, g\}$ and for some $p$, $q, r, s \in X$ and $g$ is continuous at $p, q, r$ and $s$.

(b) $g$ is $F$-weakly commuting for some $(x, y, z, w) \in C\{F, g\}$ and $g x$, $g y, g z$ and $g w$ are fixed points of $g$, that is, $g^{2} x=g x, g^{2} y=g y$, $g^{2} z=g z$ and $g^{2} w=g w$.

(c) $g$ is continuous at $x, y, z$ and $w$. $\lim _{n \rightarrow \infty} g^{n} p=x, \lim _{n \rightarrow \infty} g^{n} q=y$, $\lim _{n \rightarrow \infty} g^{n} r=z$ and $\lim _{n \rightarrow \infty} g^{n} s=w$ for some $(x, y, z, w) \in C\{F, g\}$ and for some $p, q, r, s \in X$. 
(d) $g(C\{F, g\})$ is a singleton subset of $C\{F, g\}$.

If we put $g=I$ (the identity mapping) in the Corollary 2.8, we get the following result:

Corollary 2.10. Let $(X, d)$ be a complete metric space, $F: X^{4} \rightarrow C B(X)$ be a mapping satisfying

$$
H(F(x, y, z, w), F(p, q, r, s)) \leq k \max \{d(x, p), d(y, q), d(z, r), d(w, s)\},
$$

for all $x, y, z, w, p, q, r, s \in X$, where $0<k<1$. Then $F$ has a quadruple fixed point.

If we put $g=I$ (the identity mapping) in the Corollary 2.9, we get the following result:

Corollary 2.11. Let $(X, d)$ be a complete metric space, $F: X^{4} \rightarrow C B(X)$ be a mapping satisfying

$$
H(F(x, y, z, w), F(p, q, r, s)) \leq k\left[\frac{d(x, p)+d(y, q)+d(z, r)+d(w, s)}{4}\right],
$$

for all $x, y, z, w, p, q, r, s \in X$, where $0<k<1$. Then $F$ has a quadruple fixed point.

\section{REFERENCES}

[1] H. Aydi, E. Karapinar and I. S. Yuce, Quadruple fixed point theorems in partially ordered metric spaces depending on another function, Hindawi Publishing Corporation, ISRN Applied Mathematics, Article ID 539125 (2012), 16 pages, doi: $10.5402 / 2012 / 539125$.

[2] T.G. Bhaskar and V. Lakshmikantham, Fixed point theorems in partially ordered metric spaces and applications, Nonlinear Analysis: Theory, Methods \& Applications, 65(7) (2006), 1379-1393.

[3] B. Deshpande, Common fixed point for set and single valued functions without continuity and compatibility, Mathematica Moravica, 11 (2007), 27-38.

[4] B. Deshpande and A. Handa, Quadruple fixed point theorems for multivalued mappings under $\varphi-\psi$ contraction on partially ordered metric spaces, accepted for publication in SEA Bull. Math.

[5] E. Karapinar, Quartet fixed point for nonlinear contractions, http://arxiv.org/abs/1106.5472.

[6] E. Karapinar, A new quartet fixed point theorem for nonlinear contractions, Journal of Fixed Point Theory Appl., 6(2) (2011), 119-135.

[7] E. Karapinar and N. V. Luong, Quadruple fixed point theorems for nonlinear contractions, Computer \& Mathematics with Applications, 64(6) (2012), 1839-1848.

[8] E. Karapinar, Quadruple fixed point theorems for weak contractions, ISRN Mathematical Analysis, Article ID 989423 (2011), 16.

[9] E. Karapinar and V. Berinde, Quadruple fixed point theorems for nonlinear contractions in partially ordered metric spaces, Banach J. Math. Anal., 6(1) (2011), 119-135. 
[10] I. Kubiaczyk and B. Deshpande, A common fixed point theorem for multivalued mappings through T-weak commutativity, Mathematica Moravica, 10 (2006), 55-60.

[11] I. Kubiaczyk and B. Deshpande, Common fixed point of multivalued mappings without continuity, Fasciculi Mathematici, 37(9) (2007), 19-26.

[12] V. Lakshmikantham and L. Ciric, Coupled fixed point theorems for nonlinear contractions in partially ordered metric spaces, Nonlinear Analysis: Theory, Methods \& Applications, 70(12) (2009), 4341-4349.

[13] J.T. Markin, Continuous dependence of fixed point sets, Proceedings of the American Mathematical Society, 38 (1947), 545-547.

[14] S.H. Rasouli and M. Bahrampour, On Quadruple fixed point theorems in partially ordered metric spaces, International journal of mathematics and statistics, 13(1) (2013).

[15] B. Samet and C. Vetro, Coupled fixed point, F-invariant set and fixed point of $N$ order, Ann. Funct. Anal., 1 (2010), 46-56.

[16] S. Sharma and B. Deshpande, Fixed point theorems for set and single valued mappings without continuity and compatibility, Demonstratio Mathematica, XL(3) (2007), 649658.

Bhavana Deshpande

Department of Mathematics

Govt. P. G. Arts \& Science College

RATLAM (M.P.)

INDIA

E-mail address: bhavnadeshpande@yahoo.com

\section{Amrish Handa}

Department of Mathematics

Govt. P. G. Arts \& Science College

Ratlam (M.P.)

INDIA

E-mail address: amrishhanda83@gmail.com 